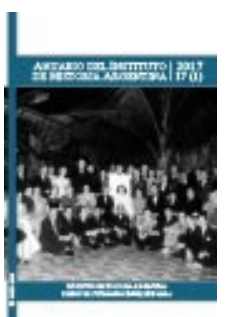

Anuario del Instituto de Historia Argentina, vol. 17, nº 1, e038, junio 2017.

ISSN 2314-257X

Universidad Nacional de La Plata.

Facultad de Humanidades y Ciencias de la Educación.

Centro de Historia Argentina y Americana

\title{
La OIT entre la dictadura y la democracia en la Argentina: aportes sobre el papel de organizaciones internacionales en la reconfiguración de las relaciones laborales en la primera mitad de los años 80
}

\author{
The ILO from dictatorship to democracy in Argentina: contributions \\ about the role of international organizations in the process of \\ reconfiguration of labor relations during the first half of the 1980s
}

\section{Victoria Basualdo *}

* Área de Economía y Tecnología de FLACSO - CONICET, Argentina | basuvic@yahoo.com.ar

\section{PALABRAS CLAVE RESUMEN}

Organizaciones del Trabajo

OIT

Dictadura militar argentina

(1976-1983)

\section{KEYWORDS}

International labor organizations

ILO

Argentine dictatorship (1976-1983)

\begin{abstract}
Este artículo tiene como objetivo analizar el papel de la Organización Internacional del Trabajo (OIT) en los años de la última dictadura en Argentina (1976-1983) y en los comienzos del gobierno democrático (1983-1985), promoviendo la investigación en mayor profundidad de la incidencia y participación de las organizaciones sindicales internacionales en la reconfiguración de las relaciones laborales y sindicales en esos años. En la primera parte se analizan algunas de las intervenciones de la OIT durante la dictadura entre 1976 y 1983, prestando particular atención a la importancia tanto del caso 842 presentado ante el Comité de Libertad Sindical, como de las conferencias anuales y diversas formas de interacción con esta organización en dicha etapa. En la segunda parte, el trabajo aborda el papel de esta organización en el contexto argentino durante los dos primeros años de la transición a la democracia (1983-1985). Aunque existen valiosos aportes sobre los intentos de replantear el funcionamiento de las organizaciones sindicales en esos años, la participación de la OIT y de otras organizaciones internacionales en las definiciones y políticas respecto al movimiento sindical está relativamente poco abordada, a pesar de ser relevante. Al mismo tiempo, el análisis de las relaciones sindicales en cada uno de estos períodos ha tendido a llevarse adelante separadamente, y proponemos aquí que ponerlos en diálogo podría abrir líneas interesantes de análisis. En el apartado final se presentan algunas conclusiones y sobre todo posibles líneas de investigación hacia adelante.
\end{abstract}




\section{La OIT y la dictadura argentina (1976-1983) $\frac{1}{}$}

La coyuntura de mediados de los años 70, y en particular el período que se abrió a partir del golpe del 24 de marzo de 1976 constituyeron un punto de inflexión significativo en la historia argentina que se caracterizó por fuertes transformaciones en la estructura económica y social. La clase trabajadora se vio afectada en este período por políticas represivas, laborales y económicas. En lo que se refiere a los profundos cambios económicos, la reforma financiera de 1977 en conjunción con la arancelaria y el endeudamiento externo, derivados de la apertura de 1979, promovieron una reestructuración regresiva del sector industrial. Ésta implicó una caída de la participación del sector industrial en el PBI, que además tuvo un impacto heterogéneo sobre las distintas ramas, y provocó un proceso de concentración y centralización económica. En este contexto, se produjo un incremento exponencial del endeudamiento externo, el cual estuvo vinculado en forma creciente con el proceso de valorización financiera (Basualdo E., 2006). Este cambio del patrón de acumulación de capital constituye el contexto imprescindible para analizar las transformaciones experimentadas por los trabajadores y sus organizaciones durante la dictadura (Basualdo V., 2010b).

Estas transformaciones estructurales se impusieron en el marco de un estado terrorista que -habiendo accedido al poder en un contexto de confrontación y violencia que según sus declaraciones fundacionales se proponía solucionar- perpetró las mayores violaciones a los derechos humanos en la historia argentina, entre las que se cuentan 30.000 desaparecidos, miles de muertos, presos, detenidos y torturados, así como exiliados y desplazados en el propio territorio, entre otros crímenes que incluyeron la supresión de la identidad de cientos de hijos de detenidos y numerosos delitos de apropiación de bienes. En este contexto, es necesario detenerse en las características de la represión a los trabajadores, y en particular a los representantes en los lugares de trabajo, que aunque no se inició con el golpe militar y tuvo hitos previos de gran relevancia, se acentuó de forma notable a partir del 24 de marzo de 1976 y experimentó a partir de entonces cambios cualitativos $\stackrel{2}{2}$. Numerosos testimonios, documentos y evidencias indican que los activistas, los delegados y los miembros de las comisiones internas estuvieron entre los blancos más buscados por las fuerzas militares, y fueron en muchos casos secuestrados, detenidos, desaparecidos o asesinados a la entrada o salida de su trabajo, o incluso en sus mismos puestos laborales (Basualdo V., 2006; Izaguirre, 2009). La evidencia sistematizada y analizada por investigadores y activistas señala que la represión a los delegados, miembros de comisiones internas y militantes, si bien estuvo dirigida y ejecutada por las fuerzas de seguridad, contó con el apoyo activo y la participación directa de funcionarios importantes de grandes empresas, que en muchos casos denunciaron a sus propios trabajadores, financiaron a las fuerzas represivas proveyéndoles fondos e infraestructura, e incluso admitieron la existencia de espacios clandestinos de detención y tortura de los trabajadores en el interior de algunas de sus plantas (Basualdo V.; 2006; Verbitsky y Bohoslavsky (2013); AEyT de FLACSO, CELS, PVJ y SDH, 2015). Un símbolo ilustrativo de esta confluencia entre la elite empresarial y las fuerzas militares, y por lo tanto de la relación estrecha entre la política económica y la violación a los derechos humanos, es la figura de José Alfredo Martínez de Hoz, quien antes de ser ministro de Economía había sido presidente del directorio de Acindar, una de las empresas en las que la represión a los trabajadores fue más aguda, desde 1975 en adelante (Basualdo V., 2006; Azpiazu, Basualdo y Schorr, 2010; Basualdo V., 2010c).

A estas formas de represión se sumó la intervención de sindicatos y federaciones, comenzando con la de la central nacional de trabajadores, la Confederación General del Trabajo (CGT). En los primeros tres años, en los que se alcanzó el punto represivo más alto, se intervinieron varias de las principales organizaciones obreras, se les colocaron interventores militares en su cúspide, y se les retiró la personería jurídica a varias de ellas, con impactos que aún se encuentran bajo estudio y debate (Zorzoli, 2015). Asimismo, la dictadura militar promovió un conjunto de normas tendientes a legalizar la actividad represiva y un creciente control y disciplinamiento en el lugar de trabajo. Desde el inicio de la dictadura se estableció el congelamiento de todo tipo de actividad gremial, así como la prohibición de toda forma de organización y protesta en el ámbito 
laboral ${ }^{3}$. La legislación estaba en estrecha relación con las medidas desarrolladas en los lugares de trabajo por lo cual, a medida que los trabajadores encontraban o creaban formas no prohibidas de organización o manifestación, las mismas iban siendo contrarrestadas por normas posteriores.

Entre 1976 y 1983 la OIT había adquirido un papel significativo en al menos dos sentidos principales. Por un lado, recibió y evaluó casos denunciando la violación de derechos sindicales durante la dictadura. Inmediatamente después del golpe militar, la Federación Sindical Mundial (FSM), la Confederación Mundial del Trabajo (CMT), junto a otras confederaciones y organizaciones nacionales como la Confederación de Trabajadores de Perú, presentaron quejas ante el Comité de Libertad Sindical de la OIT que se concentraron en el caso 842, el cual se convirtió en el caso más importante referido a Argentina. Este caso no constituyó una novedad completa. En los años previos al golpe militar del 24 de marzo de 1976, en un contexto en que los procesos de movilización obrera y también de represión de la protesta habían ido en aumento -desde fines de los años 60 y durante la primera mitad de los años 70-, el Comité de Libertad Sindical había recibido varias denuncias referidas a la situación en Argentina, que se transformaron en casos. Algunos ejemplos fueron el caso 809 iniciado en 1974 por la Federación Gráfica Bonaerense -que denunciaba la quita de su personería gremial y la persecución y encarcelamiento de varios de sus líderes-, y el caso 836 -abierto por la Confederación Mundial del Trabajo en enero de 1976 contra el gobierno de Argentina- que denunciaba la permanente interferencia con el ejercicio de los derechos sindicales en Argentina ${ }^{4}$.

El caso 842 se refería a dos cuestiones principales: la detención y desaparición de líderes sindicales, militantes y trabajadores, y la violación de los derechos sindicales básicos en Argentina, lo cual incluía la intervención de los principales sindicatos, de la CGT, y también todas las restricciones impuestas a la actividad sindical y a los derechos laborales ${ }^{\underline{5}}$. Aun cuando había tenido otros precedentes como los mencionados, el caso 842, iniciado muy poco tiempo después del golpe de estado -cuando ya era visible el fuerte carácter represivo que adquiría la dictadura sobre los trabajadores y sus organizaciones-, adquirió una importancia y un desarrollo central a partir de la incorporación periódica de información y de denuncias no sólo de las organizaciones que lo habían iniciado, sino de otras, como la Confederación Internacional de Organizaciones Sindicales Libres (CIOSL) y otras confederaciones nacionales. Además, organizaciones de varios países se sumaron a apoyarlo, sostenerlo y nutrirlo de información nueva.

Más allá de las discusiones de este caso en el marco del Comité de Libertad Sindical, la conferencia anual de la OIT en Ginebra se convirtió también en un hecho político de mucha importancia que atrajo la atención de la prensa, la cual publicaba año a año tanto artículos referidos a la composición de la delegación como referencias diversas al caso argentino. Más allá de la llamativa atención que la prensa nacional dedicó a los encuentros anuales, había diversas publicaciones referidas a la situación de la OIT en sí misma, ya que la organización se encontraba en una situación compleja por la partida anunciada de Estados Unidos de dicha organización $\underline{6}$.

Estudios previos realizados que analizan pormenorizadamente estos dos aspectos permiten, por un lado, ver que la OIT cumplió un papel importante durante la última dictadura argentina en varios sentidos. Estas investigaciones muestran que la disputa en torno a la imagen internacional resultaba de gran importancia para la dictadura, que prestaba considerable atención a la forma en la que era vista en esferas internacionales, por razones políticas, diplomáticas y comerciales. En lo que se refiere a la conferencia anual de la OIT, la dirigencia dictatorial, preocupada por la extensión e impacto de la campaña de denuncia internacional, consideró importante intentar garantizar una presencia tripartita que pudiera dar una imagen exterior de normalidad de las relaciones sociales y económicas del país, y de legitimidad de las autoridades militares. Esto ocurrió en varias ocasiones, aunque en otras, como las de 1977 y 1980, no pudo concretarse y sólo asistió un único representante de la dictadura. En un contexto de censura, control sobre la prensa y represión 
intensa, encuentros internacionales como la conferencia anual eran también foros de importancia potencial central para difundir lo que ocurría en la Argentina. Precisamente por esta cuestión se convirtió también en un eje importante de controversia y discusión en el seno del movimiento sindical, y en un objetivo para los sectores perseguidos que buscaban también lograr alguna presencia allí aunque fuera informal.

Por su parte, el análisis del caso 842 llevado adelante en el marco del Comité de Libertad Sindical provee evidencia interesante para repensar la potencialidad y también las limitaciones de este tipo de instancias internacionales. Por un lado, la presión impuesta sobre la dictadura argentina tuvo efectos como la liberación de algunos de los líderes que habían sido denunciados en las listas de detenidos y perseguidos, y que fueron liberados para poder mostrar avances por parte de la dictadura. Por otro lado, la estrategia que adoptó la dictadura argentina de permanente y cordial respuesta a las solicitudes y aparente colaboración con las investigaciones consistió en contestar puntualmente a todas las demandas, satisfaciendo las instancias formales. Esto llevó a estos funcionarios a diferenciar el caso de Argentina de los de Chile o Uruguay, que habían atraído considerablemente mayor atención y rechazo internacional. La presencia de los representantes de la Junta Militar y su aparente compromiso para colaborar -y proveer explicaciones y justificaciones para cada una de las denuncias y demandas- tuvieron éxito en demorar la reacción de la OIT y en disminuir muy fuertemente sus alcances, debido a que lograron formatos de intervención que frenaron el impacto de las decisiones, pronunciamientos y acciones de la organización respecto de la dictadura.

En suma, el análisis del proceso complejo de la acción de la OIT en este tiempo permite ilustrar algunas de las potencialidades y también las limitaciones de las instancias de organización internacional en un tiempo de grandes transformaciones estructurales y ante la violación masiva de derechos humanos y laborales. Investigaciones recientes echan luz también sobre distintas formas de participación en la OIT de sectores sindicales que, lejos de buscar la denuncia de la dictadura, convivieron con ella o incluso legitimaron su accionar (Zorzoli, 2016).

Si por un lado los elementos desarrollados permiten identificar impactos positivos de la acción en este organismo -que incluyen cuestiones relevantes como la verificación y acreditación de muchas de las violaciones denunciadas por distintas organizaciones nacionales e internacionales, así como distintas formas de presión sobre las autoridades que pueden haber tenido efectos y resultados en la liberación de presos y el reconocimiento de algunos derechos a los que no fueron liberados-, por otro, también pone de manifiesto las limitaciones, demoras y dificultades que tuvo para operar sobre estos procesos, particularmente en casos en los que el gobierno dio respuestas formales que cumplían con lo solicitado, logrando ocultar o disfrazar algunas de las violaciones más flagrantes. Todo esto plantea la necesidad de profundizar el análisis de las complejas interacciones en el seno de la OIT, así como de las funciones posibles de la acción sindical internacional.

\section{La OIT, el gobierno y el movimiento sindical argentino en los primeros años de democracia (1983- 1985)}

A partir de esta breve y limitada síntesis sobre algunas dimensiones del papel que desempeñó la OIT como organismo internacional -en un tiempo particularmente crítico en lo que se refiere a violación de derechos humanos, laborales y sindicales, el cual se convirtió en campo de disputa entre sectores con visiones distintas de la dictadura- este segundo apartado tiene por objetivo contribuir a comprender cuál fue su presencia e impacto en el comienzo del gobierno democrático encabezado por el presidente Raúl Alfonsín, quien asumió funciones el 10 de diciembre de 1983. En primer lugar, se analiza la perspectiva y propuestas del nuevo gobierno sobre las relaciones laborales y las organizaciones sindicales, particularmente el proyecto conocido como Ley Mucci que estuvo en el centro del debate político, económico y social. En segundo lugar, se 
aportan evidencias sobre la interpelación y la intervención de las organizaciones sindicales internacionales y particularmente de la OIT, que tuvieron, hasta ahora, escaso lugar en los análisis existentes. En tercer lugar, se analiza muy brevemente el fracaso en la aprobación del proyecto de ley mencionado, y la aprobación de la ley 23.071 en julio de 1984. Finalmente, se aportan algunas evidencias adicionales sobre el papel de funcionarios y dependencias de OIT en este proceso denominado de normalización sindical hasta 1985.

Una de las razones por las que ha resultado difícil incluir estas dimensiones en el análisis es la inaccesibilidad de las fuentes de archivo, debida a una ineficiente tradición de conservación y acceso a la documentación por parte de las organizaciones sindicales y dependencias estatales en Argentina, y a restricciones muy fuertes de acceso a las existentes, tanto por parte de las organizaciones sindicales como del estado. Al mismo tiempo, el archivo de la OIT, altamente organizado y sistematizado, tiene reglas de protección de su documentación interna de archivo por extensos períodos de entre 30 y 50 años, lo que obstaculizó el acceso considerablemente. El análisis que sigue se realizó a partir de fuentes de prensa, y también de nuevas fuentes recientemente desclasificadas provenientes del Archivo de la Organización Internacional del Trabajo en Ginebra que permiten reconstruir, al menos en forma parcial y preliminar, aspectos importantes de esta historia y plantear líneas posibles de análisis hacia adelante.

Las alternativas de política estatal respecto a la estructura y dinámica del movimiento sindical no resultaban sencillas en los años 80. Como el breve análisis de la etapa anterior permite apreciar, el legado dictatorial incluía cuestiones extremadamente complejas tanto en términos de las transformaciones de la estructura socioeconómica, las violaciones a los derechos humanos y el cercenamiento de derechos en una gran cantidad de ámbitos de la vida política y social ${ }^{7}$. En este marco, la cuestión de las relaciones laborales y la organización sindical era considerada tan significativa por el nuevo gobierno que a sólo seis días de haber asumido, el 16 de diciembre de 1983, se presentó en el Congreso un proyecto de reordenamiento sindical que apuntaba a garantizar la convocatoria a comicios gremiales con un régimen electoral especialmente diseñado para democratizar los mecanismos de acceso al control de dichas organizaciones. Para ello, procuraba valerse de una eventual intervención del poder estatal ejercido en el interior mismo de las entidades laborales. En síntesis, el proyecto proponía en su artículo 1 la renovación de la totalidad de los cargos sindicales mediante la convocatoria desde el Ministerio de Trabajo a elecciones de delegados y comisiones internas; la representación de minorías mediante su inclusión en los órganos de conducción cuando alcanzaren un 25\% de los votos (art. 13); y establecía que, en tales casos, dichos órganos deberían tener un tercio de representantes por la minoría. Finalmente, sostenía que la participación estatal en las elecciones internas se haría a través de la intervención de la Justicia Nacional Electoral, para garantizar que fueran procesos limpios. El Juez Electoral podría designar a uno o más veedores judiciales como contralores del acto eleccionario, por pedido de los electores, de las listas, del sindicato o del Ministerio (art. 31).

Existen varios análisis acerca de la presentación del proyecto de ley conocido popularmente como Ley Mucci, en reconocimiento al Ministro de Trabajo, Antonio Mucci, que la impulsó (Gaudio y Domeniconi, 1986; Aruguete, 2006; Gordillo, 2013). Varios de los estudios disponibles, sin embargo, abordan o bien el papel del gobierno en este proceso, o bien el papel de la cúpula del movimiento sindical, enfatizando que esto ocurrió en un marco en el que los principales dirigentes, identificados con el peronismo, habían sido debilitados por el fracaso de esta fuerza política en las elecciones nacionales del 30 de octubre, resultado respecto del que varios sectores consideraban que habían tenido una gran cuota de responsabilidad. Desde esta perspectiva, en tales circunstancias, el desafío que debían enfrentar estos dirigentes era doble. Por un lado, la decisión del gobierno ponía a prueba su capacidad de respuesta en un momento en que el tradicional poder social y político de los sindicatos se hallaba bajo la amenaza del debilitamiento y la fragmentación; por el otro, la exigencia de reafirmar su inserción en el sistema sociopolítico del país, en tanto la ofensiva oficial llevaba implícitamente el cuestionamiento a la identificación de sus estructuras organizativas con una 
determinada fuerza política, fenómeno basado en la tradicional orientación unificada de la clase trabajadora en favor del peronismo.

Una muy breve revisión de la cobertura de medios de prensa sistematizados en el archivo de la OIT revela el amplio interés que estos temas despertaban a inicios de 1984, así como la extensión y el eje predominante de la misma: una generalizada posición crítica a los principales líderes sindicales, en su mayoría afiliados al peronismo ortodoxo. Algunos ejemplos resultan útiles para analizar estas caracterizaciones:

Los dirigentes peronistas que rechazan el anteproyecto, a discutirse estos días en la Cámara de Diputados, todavía no han presentado argumentos consistentes (...) a duras penas está[n] tratando, desde la derrota electoral, de asimilar que la democracia es conciliación, convivencia y respeto a las minorías. No existe otra razón para explicar que esos dirigentes entiendan toda política orientada a disminuir el poder de las organizaciones consideradas representativas de los sectores o clases como una política coercitiva. $\underline{8}$

El diario La Nación realizó una amplia cobertura que proponía una visión de las corrientes en conflicto:

El debate surgido por el proyecto gubernamental de reordenamiento gremial muestra un atípico panorama sindical que ha desembocado en el realineamiento de los dirigentes del movimiento obrero. Dos polos bien definidos se aprestan a librar una dura batalla. Por un lado, lo que se denomina la vieja guardia del sindicalismo, en su casi totalidad peronista, que acaba de agruparse en una sola CGT. Por otro, la vigorosa aparición de nucleamientos paralelos que buscan aceleradamente espacio para insertarse en el proceso de democratización gremial que impulsa el gobierno radical a través de aquella iniciativa.

Nuevamente la caracterización de los sectores dirigentes del sindicalismo enfatiza sus rasgos conservadores en función de la preservación de sus lógicas y posiciones:

El plenario unificador realizado la semana pasada se caracterizó por su fuerte contenido antigubernamental. Esta vieja guardia gremial no trepidó en denunciar una supuesta injerencia oficial en el proceso de reorganización sindical. Detrás de esa acusación, tal vez se esconde la preocupación de los dirigentes ante la perspectiva del desplazamiento a la hora de revalidar los títulos. $\underline{9}$

La oposición y crítica a la dirigencia de la CGT era tal fuerza que llevó al diario La Nación a destacar el fortalecimiento de sectores más críticos a los que históricamente había denunciado como radicalizados, y cuyo avance había lamentado:

La puja entre el sindicalismo de la vieja guardia y los nuevos grupos sindicales ha tenido sus dos primeras manifestaciones. Alberto Piccinini, alineado en el Movimiento Nacional de Renovación Sindical e integrante del ENTRA que conduce, entre otros, Julio Guillán de (telefónicos), obtuvo un significativo triunfo en las elecciones de la seccional Villa Constitución de la Unión Obrera Metalúrgica. Su victoria, lograda con amplitud, significó un rotundo pronunciamiento contra el "miguelismo" dentro de ese gremio y se supone que hará variar la situación interna de la UOM. Los observadores computan también que el triunfo de Piccinini favorece el planteo de las seccionales de la UOM que piden el alejamiento de Miguel, cuyo predicamento declinó notablemente después del fracaso electoral del peronismo. $\underline{10}$

Al tiempo que destaca los avances de sectores críticos al liderazgo vigente en la CGT, profundiza su posición crítica respecto a los sectores dirigentes de dicha central: 
Queda sin embargo un sector de indudable peso que no termina de comprender esa nueva realidad y sigue actuando como lo hizo durante cuarenta años: se trata de la vieja dirigencia sindical que siempre se sintió en el poder y que no se ubica, ahora, frente a las circunstancias diferentes en las que debe moverse. (...) Probablemente, sin embargo, haya una razón que explique el fenómeno: esta dirigencia sindical de larga experiencia como oficialista, y en todo caso habituada a maniobrar entre bambalinas con gobiernos militares, se encuentra por vez primera con una lucha política planteada a la luz del día y en el marco del orden constitucional. $\underline{11}$

Llama la atención, entonces, en estas coberturas de los medios, el foco en las corrientes sindicales y su poder relativo, que parece partir de posiciones fuertemente críticas del movimiento sindical en términos amplios, y proponer una lectura con una preocupación política subyacente (es decir, evaluando el impacto que tendría el desplazamiento de determinados sectores en el fortalecimiento o debilitamiento del Partido Justicialista y la UCR). Es notable la exclusión de reflexiones en torno a los desafíos estructurales que habían dejado las políticas dictatoriales en los campos económicos, laborales y represivos respecto a los trabajadores en su conjunto, y a los impactos de las cuestiones de debate en términos de las posibilidades de recuperación de derechos.

En febrero de 1984 comenzó a hacerse visible en los medios de prensa lo que se convirtió en los tiempos posteriores uno de los ejes centrales: la apelación a instancias internacionales y particularmente a la OIT. El 4 de febrero de 1984 se informa que "funcionarios de la delegación Argentina se reunieron en Caracas con el titular de la OIT, Francis Blanchard, para explicar la filosofía y las propuestas que animan a la Ley de Reordenamiento Sindical. Se descuenta una futura reunión con la participación de Alfonsín, ahora ocupado

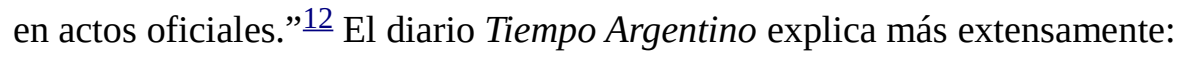

La conflictiva situación política surgida en la Argentina a partir de la iniciativa oficial de reordenar la vida sindical del país, fue objeto ayer de un minucioso análisis por parte de funcionarios del máximo nivel del gobierno de Raúl Alfonsín en el curso de una reunión que mantuvieron con el director de la Organización Internacional del Trabajo, Francis Blanchard, quien también asiste a las ceremonias de transmisión del mando presidencial en Caracas. De esta reunión participaron, además de Blanchard, su jefe de gabinete, el brasileño Wilson dos Santos y por la argentina el subsecretario de Relaciones Económicas Internacionales, Jorge Romero, y los asesores presidenciales Jorge Malamud y Jorge Sábato. $\underline{13}$

Los documentos provenientes del archivo de la OIT permiten confirmar esta información. Documentos de archivo muestran que el 15 de febrero de 1984, el presidente Raúl Alfonsín se dirigió al director general de la OIT indicando que

...el viaje del señor canciller de mi país Dante Caputo me brinda la enorme satisfacción de hacerle llegar mi cordial recuerdo e invitarlo a visitar la República Argentina en la fecha que estime más conveniente. Espero tener el gusto de verlo muy pronto, seguro como estoy de la identidad de nuestros esfuerzos por promover la consideración de la problemática del trabajo como medio de lograr una mayor justicia social, acorde con los altos fines de esa entidad. $\underline{14}$

Blanchard responde la carta el 2 de marzo de 1984, afirmando que

...es para mí un motivo de profunda satisfacción, como Director General de la Oficina Internacional del Trabajo, aceptar tan amable invitación que me permitirá estrechar los fructuosos lazos de colaboración ya existentes entre la República Argentina y esta Organización, en pro de la paz y la justicia social... 15 
Luego propone como fecha posible para el encuentro fines de septiembre u octubre de 1984. En seguimiento de esta intención de consulta, el 27 de febrero el canciller Dante Caputo hizo una exposición ante la Comisión de Derechos Humanos de las Naciones Unidas, y se reunió con el Director General de la OIT, Francis Blanchard, para discutir temas laborales $\underline{16}$.

A comienzos de marzo de 1984 tuvo lugar un nombramiento que puede resultar significativo para evidenciar el tipo de vínculos de la OIT con sectores del sindicalismo argentino: el nombramiento de Ramón Antonio Baldassini, entonces Secretario General de la Federación de Obreros y Empleados de Correos, como miembro titular de la Comisión Paritaria del Servicio de Comunicaciones Mundiales de la Organización Internacional del Trabajo. Es interesante analizar la intensa trayectoria de este sindicalista que ya en esos años había desempeñado una gran cantidad de puestos nacionales e internacionales a la luz de su posicionamiento respecto a las violaciones de los derechos de los trabajadores y sus organizaciones $\frac{17}{}$. Su posición quedó pública y claramente establecida cuando fue citado, en 1985, junto con Jorge Triaca, a declarar en la primera audiencia del Juicio a las Juntas Militares el 24 de abril de 1985. En las audiencias orales ante la Cámara Federal, Jorge Triaca destacó el buen trato que habría recibido de los militares durante su prisión, mientras que Ramón Baldassini manifestó no conocer casos de sindicalistas desaparecidos o asesinados durante la dictadura militar, y rememoró en cambio los asesinatos de los dirigentes de la CGT presumiblemente cometidos por la guerrilla en los años previos (Galante, 2015). De acuerdo con contribuciones relevantes, lejos de representar una postura generalizada, estas declaraciones causaron una gran conmoción en el ámbito gremial, ya que varios representantes de los trabajadores habían apoyado públicamente el juicio en los meses previos (Galante, 2015). El sindicato de Luz y Fuerza y la propia CGT, dos de cuyos escaños en la Secretaría General eran ocupados por Baldassini y Triaca, habían firmado conjuntamente una solicitada que salió publicada a página completa en Clarín del 11 de febrero, al cumplirse el aniversario del secuestro y asesinato del entonces Secretario General de Luz y Fuerza, Oscar Smith, en 1977, en el que reclamaban una "respuesta de la democracia" por su desaparición. Tras los testimonios de Triaca y Baldassini del 24 de abril las entidades de derechos humanos remitieron a Triaca un largo listado de sindicalistas desaparecidos, y dos días más tarde, la "Comisión de los 25" de la CGT, aliada al peronismo renovador e integrada entre otros por el cervecero Saúl Ubaldini, José Rodríguez de SMATA, Osvaldo Borda del gremio del caucho y el ferroviario Pedraza, pidió la renuncia o destitución de Baldassini, cuestión que no se concretó por el apoyo del sector "independiente", y el de figuras como la del propio Triaca (Galante, 2015). El nombramiento de Ramón Baldassini en forma permanente en la OIT -nombramiento que de acuerdo a fuentes de prensa, él consideró como "un honor para todo el movimiento obrero argentino y un reconocimiento a sus luchas y a su organización”, para luego afirmar: "comprometo todo mi esfuerzo en no defraudar a los trabajadores argentinos en este nuevo puesto de lucha"- fue mantenido luego de estos sucesos en 1985, lo cual constituye un dato relevante respecto de la importancia dada a los posicionamientos sobre las violaciones de derechos humanos y sindicales en la agenda de la OIT $\underline{18}$.

Adicionalmente, muy pocos días antes de la votación del proyecto de ley en el Congreso, una misión de la CIOSL arribó a la Argentina y recibió una gran cobertura de la prensa e interés en círculos políticos y sindicales. Medios de prensa informaron sobre críticas de esta delegación al proyecto de ley, afirmando que

...el coordinador de la Confederación Internacional de Organizaciones Sindicales Libres (CIOSL), Enzo Friso, criticó aspectos de la ley de reordenamiento sindical que se debate en el Parlamento argentino. Una delegación de la CIOSL, de orientación social-demócrata, arribó ayer por la mañana a Argentina, para sostener sucesivas reuniones con sindicalistas argentinos, chilenos y paraguayos siendo recibida por el dirigente de la CGT unificada, Saúl Ubaldini. La comitiva está integrada por Enzo Friso (Italia), Luis Anderson (Estados Unidos), Manuel Simón (España), William Doherty (Estados Unidos), y Luigi Cal (Italia), entre otros. En declaraciones formuladas en el aeropuerto de 
Ezeiza, Friso dijo conocer la ley de reordenamiento sindical y destacó que algunas de sus normas entran en conflicto con lo dispuesto en la Carta de la Organización Internacional del Trabajo. En este sentido afirmó que en los países verdaderamente democráticos "no es una ley la que debe resolver los problemas sindicales, sino que son los estatutos de los gremios los que deben tener primacía”. Precisó que la parte de la ley que limita el tiempo de los mandatos de los dirigentes sindicales "es contraria al derecho de los trabajadores de elegir a quien quieren y por el tiempo que sea necesario.

Ese es un problema interno de cada gremio y el estado no tiene por qué intervenir”. $\underline{19}$

También en el diario Tiempo Argentino se destaca, el martes 13 de marzo de 1984 que "El gremialismo continental apoyó la posición de la CGT. En diálogo con el Presidente, sugirieron que se apliquen los convenios suscriptos en la OIT”. En el análisis, se sostiene que

...la delegación de la Central Internacional de Confederaciones Sindicales Libres, que visita nuestro país, se entrevistó ayer con el Presidente Raúl Alfonsín y le recomendó “actuar con calma” en lo que hace a la ley de reordenamiento sindical. El grupo de dirigentes de la CIOSL -una de las principales centrales obreras internacionales, de tendencia social-demócrata- llegó al despacho presidencial encabezada por Enzo Friso, y allí los estaba esperando el primer mandatario junto al Secretario General de la Presidencia, Germán López, y al Ministro de Trabajo, Antonio Mucci. Friso, en nombre de los visitantes, informó luego de la reunión que la CIOSL “ofreció al gobierno la ayuda de los expertos de la Organización Internacional del Trabajo para evitar que se tomen decisiones que entren en conflicto con los convenios internacionales y que obliguen a la CIOSL a tomar cartas en el asunto".

Respecto de los sectores que motorizaron esta presencia, se enfatizaba que la convocatoria había estado a cargo de sectores dirigentes de la CGT, que apoyaron fuertemente sus intervenciones:

Fuentes gremiales confirmaron ayer que la audiencia de la CIOSL con el Presidente Alfonsín fue gestionada por la central obrera argentina. Esos dirigentes, todos miembros del consejo directivo cegetista, no ocultaban ayer su satisfacción por la gestión de los hombres de la central internacional en nuestro país y destacaban "la importancia” de su pronunciamiento. $\underline{21}$

El 15 de marzo de 1984, finalmente, se produjo la derrota del proyecto de ley en la sesión del Senado por 24 votos contra 22. La cobertura de prensa indica que, al cabo de diez horas de discusión, el Senado desechó la sanción de Diputados al proyecto de ley de reordenamiento sindical con el voto justicialista, que se sumó al del MID y del Movimiento Popular Neuquino. La sesión del Senado resultó la más extensa del período extraordinario hasta ese momento. La derrota de este proyecto de ley tuvo un muy considerable impacto político, y recibió una amplísima cobertura de prensa y atención en el debate público, ya que definía límites y condicionamientos claros para un gobierno que intentaba consolidar su autoridad y fortaleza $\underline{22}$.

Diversas contribuciones intentaron explicar lo que fue considerado como un fracaso en la democratización sindical al no haber podido el gobierno imponer su proyecto. En general parece haberse partido de la idea de que Alfonsín tenía un proyecto democratizador que no pudo poner en práctica por la presión negativa de las corporaciones sindicales que, tanto en éste como en muchos otros aspectos, ejercieron su poder, lo que forzó a la UCR a ceder para mantener la gobernabilidad. Al tiempo que Roberto Gargarella (2010) subrayó que Alfonsín creía que bastaba con otorgar derechos “desde arriba” e instalar los temas en la agenda para esperar respuestas positivas posteriores, lo que a su juicio implicaba una subestimación del impacto social de la dictadura, Murillo (2010) destacó que con el proyecto Mucci el Presidente Alfonsín habría intentado hacer valer el peso de los votos frente a las corporaciones lanzando una estrategia de confrontación que, luego, 
debió cambiar por otra de concertación y de otorgamiento de recursos institucionales para poder avanzar en la aplicación de sus políticas económicas. Sin embargo, otras contribuciones como las de Gaudio y Domeniconi (1986), centradas en el análisis de las elecciones sindicales que tuvieron lugar a partir de la sanción de la ley $\mathrm{N}^{\circ} 23.071$ en julio de 1984, mostraron que, a pesar de la normativa sancionada en contra de las aspiraciones del gobierno, se produjeron cambios significativos hacia una mayor competencia y participación en un sentido democratizador en varios sindicatos. Este y otros trabajos como los de Palomino (2005) enfatizan la heterogeneidad sindical, escasamente abordada en el discurso oficial y en gran parte de la producción académica sobre el período, y plantean la necesidad de analizar los sentidos y alcances de la democratización, que no era una aspiración exclusiva del gobierno. Contribuciones recientes de Mónica Gordillo (2013) siguen esta línea interpretativa, y han cuestionado la visión de Alfonsín como defensor ejemplar del proyecto de democratización sindical al que se contraponía la propuesta de normalización sostenida por los sindicatos, interpretada como mera restauración del autoritarismo del pasado. De acuerdo a Gordillo,

...lo anterior ocultaba una falsa disyuntiva dado que, desde las expectativas de la mayoría de las organizaciones sindicales, un proceso genuino de democratización debía partir de restituir los derechos sindicales que habían sido derogados; así, desde esta perspectiva, más que propuestas antagónicas podían considerarse complementarias o, en todo caso, sucesivas. Por otra parte, poco se reflexionó sobre los contenidos dados a la democratización por los distintos sectores sindicales y sobre los mecanismos de interacción entre los actores y el gobierno -esenciales en todo proceso democratizador-, entre otras cuestiones que se relacionan con el papel del Estado en la consolidación democrática (Gordillo, 2013, p. 145).

Todas estas perspectivas convocan a un análisis más complejo y cuidadoso del estado del movimiento sindical luego de la dictadura, que debe detenerse en la distinta evolución e impacto de las reformas estructurales en los distintos sectores, y distinguir situaciones diferenciales.

Más allá de los debates, es claro que el fracaso del proyecto de ley modificó la situación en la cual se pudo desarrollar el proceso de "normalización” sindical. En julio de 1984 el Congreso aprobó un reglamento electoral, la ley 23.071, cuyo contenido, acordado con los mismos dirigentes sindicales, constituyó una implícita renuncia a varios de los objetivos que se había fijado el gobierno radical al principio de su gestión, en particular, su intención original dirigida a refundar el movimiento sindical. De todos modos, el presupuesto político del nuevo marco legal fue propiciar los mínimos requisitos del juego democrático en los procesos eleccionarios sin perjuicio del reconocimiento de la autonomía de las organizaciones sindicales. Sin embargo, el proceso eleccionario desarrollado dentro del nuevo marco legal mostró en los hechos algunos cambios relevantes respecto de los rasgos que habían caracterizado durante décadas los comicios sindicales en el país, e introdujo, a la vez, cierta variación significativa en la conformación del movimiento sindical como actor social y político.

Estas definiciones, lejos de atenuar los vínculos y la importancia otorgada a la OIT, fueron la antesala de sucesivos contactos con representantes de esta organización. El 13 de abril de 1984 Julio Galer, entonces director general adjunto de la OIT, informa en un cable urgente y personal que después de un encuentro con el ministro de trabajo, el ministro de relaciones exteriores, trabajadores y empleadores terminó su misión con un encuentro con el presidente de la nación, Raúl Alfonsín, quien recibió el mensaje y requirió en forma oficial una misión de alto nivel de la OIT para proporcionar asesoramiento a la elaboración de una futura legislación sobre asociaciones profesionales y libertad de asociación indicando, además, que resultaba imperativo que al menos la primera fase de la misión se llevara adelante en el mes de mayo $\underline{23}$. 
Esta cuestión es retomada en un cable del director general de la OIT, Francis Blanchard, a Raúl Alfonsín con fecha 2 de mayo de 1984 en la que expresa:

Particularmente grato manifestar a Ud. mi satisfacción por la excelente acogida que su gobierno brindó a mi colega y amigo Julio Galer. Muy complacido por la total identidad de nuestros puntos de vista en materia social. Dando cumplimiento a la honrosa solicitud presentada por el Superior Gobierno puedo confirmar eminente jurista Profesor Nicolás Valticos, griego, largo tiempo jefe del Departamento de Normas de la OIT y líder en importantes misiones internacionales. Llegará a Argentina el 27 de mayo. A fin de realizar trabajos preparatorios llegará a Buenos Aires el 20 de mayo Bernard Gernigon, francés, jurista laboral, actualmente alto funcionario de Normas OIT. Desde ya agradezco a Ud. todo cuanto pueda facilitar a mis representantes personales. $\underline{24}$

Esta visita tuvo nuevamente una gran cobertura y repercusión en medios de comunicación que desde mediados de mayo de 1984 se refieren al inminente arribo de los funcionarios de la OIT, para dar inicio a "los trabajos preparatorios de la misión de la Organización Internacional del Trabajo, que tiene por objeto asesorar al gobierno argentino en materia de reordenamiento de los aspectos jurídicos de la normalización sindical”르. Se refieren al arribo de Bernard Gernigon, jefe adjunto del Servicio de Libertad Sindical de la OIT, quien precedió al Dr. Nicolas Valticos, líder de la misión principal a Buenos Aires.

Ambas etapas de la misión incluyen encuentros muy significativos con diferentes funcionarios estatales y representantes sindicales y de empleadores. Particularmente importante fue la reunión del jefe adjunto del Servicio de Libertad Sindical de la OIT, Bernardo Gernigon, con el entonces Ministro de Trabajo Juan Manuel Casella $\underline{26}$. El diario La Razón resalta, por su parte, que

...la prioridad que el gobierno le está otorgando al proceso de normalización gremial sigue de manifiesto en el marco de consultas y entrevistas de alto nivel como por ejemplo, tras la ajetreada jornada del martes, el encuentro que tuvieron en la tarde de ayer, en Olivos, el presidente y su Ministro de Trabajo. $\underline{\underline{27}}$

La cobertura de prensa de la misión encabezada por Valticos fue muy amplia y reveladora de la gran importancia que adquirió la presencia de los delegados de la OIT en un momento clave de definiciones sobre el movimiento sindical y el reordenamiento de las relaciones laborales. De los documentos internos de la misión de Valticos disponibles en el archivo resulta particularmente notable la síntesis preparada sobre la evolución histórica del sindicalismo argentino. Prácticamente no hay una evaluación de las políticas de la dictadura y sus impactos, ni de los desafíos que éstas conllevaban para ese tiempo. En el informe de síntesis sobre las etapas de la historia argentina, sólo se afirma que el tiempo entre 1973 y 1976 se caracterizó por convulsiones políticas, creciente violencia, crisis económica y agudas confrontaciones dentro del campo del trabajo y del partido peronista. Indican que en 1976 las fuerzas militares tomaron el poder y la CGT fue prohibida. "Casildo Herreras y otros líderes sindicales debieron partir al exilio, Lorenzo Miguel y otros fueron a prisión, y muchos políticos y líderes sindicales fueron asesinados o desaparecidos”. Resulta notable -luego de acreditar la magnitud de la información disponible en el propio archivo de la OIT, la cual fue procesada en distintas instancias de esta organización a lo largo de los años dictatoriales- la ausencia total de discusión sobre los impactos amplios de la dictadura tanto en términos de las condiciones de los trabajadores como del funcionamiento de la estructura sindical. Esto implicaba en los hechos una ausencia total de diagnóstico sobre los derechos perdidos, y las complejas vinculaciones entre trabajadores, sindicatos, poder económico y Estado $\underline{28}$.

Es también relevante el análisis incluido en el informe de la misión sobre las relaciones entre las distintas corrientes sindicales. Se destaca muy especialmente que la vinculación de todos los sectores (incluyendo a la 
CGT, los 20, y Renovación y cambio) con la OIT son excelentes. Se enfatizan también los vínculos de la jerarquía eclesiástica con dirigentes como Saúl Ubaldini y José Rodríguez, quien es presentado además como el representante del así llamado Movimiento de Unidad Latinoamericana, creado y respaldado por la CLAT. Los contactos sugeridos para concretar reuniones e instancias de trabajo con el movimiento sindical incluyeron nuevamente a Ramón Baldassini -de quien se destacaba que era miembro de la comisión de gobierno de la OIT y uno de los cuatro secretarios generales en la presente estructura de la CGT-, y a Jorge Triaca, Saúl Ubaldini y Osvaldo Borda -de quienes se destacaba que eran los otros tres secretarios generales en la presente estructura-, junto a Juan Racchini y Enrique Micó del grupo de los 20 (Barrionuevo, parte también de este grupo, era en ese momento Ministro de Trabajo), Juan José Zanola (bancarios) y Ongaro (gráficos), ambos considerados independientes $\underline{29}$.

La síntesis proporcionada por el diario Tiempo Argentino resume adecuadamente los apuntes disponibles de Valticos al final de su misión. La nota afirma que

...el enviado especial de la OIT para asesorar al gobierno argentino en legislación sindical, Nicolás Valticos, dijo ayer que "son los propios trabajadores los que deben decidir si quieren sindicatos unitarios o pluralistas” y opinó que la relación actual entre las autoridades y la dirigencia gremial se caracteriza por la búsqueda de “una clara concertación”. $\underline{30}$

El visitante formuló estas opiniones después de reunirse con el presidente Raúl Alfonsín en la Casa de Gobierno, en un encuentro en el que también participó el ministro de Trabajo Juan Casella y el jefe adjunto de la Comisión de Libertad Sindical de la OIT, Bernard Gernigón.

No es un tema tan sencillo; desde el punto de vista de la OIT el gobierno debe permitir un sindicalismo libre, no debe intervenir en su vida cotidiana, no tiene que imponer una u otra forma de sindicalismo y debe permitir a los sindicatos, si ellos lo desean, un sindicalismo pluralista, pero su forma debe ser definida por los sindicatos (...) los trabajadores deben decidir sobre sus actividades y el gobierno puede disponer dentro de la ley algunos principios que deben regir el funcionamiento democrático del movimiento sindical. $\underline{\underline{31}}$

Esta presencia y vinculaciones tuvieron continuidad tanto en instancias técnicas como en contactos sostenidos entre las máximas autoridades. En el primer sentido, se destaca la visita de funcionarios de la OIT -5 al 9 de noviembre de 1984- en el marco de un proyecto de investigación dirigido por Raffaele de Grazia cuyo objetivo central de era realizar una cantidad de entrevistas con dirigentes argentinos particularmente activos en el campo de los servicios sociales, lo que incluyó reuniones de trabajo enfocadas en el análisis del papel y alcance de las obras sociales sindicales $\underline{32}$. En cuanto a vinculaciones de funcionarios de primer nivel, se destaca una nueva misión de la OIT a Argentina, esta vez encabezada por el propio director general de la organización, Francis Blanchard, en abril de 1985. Esta visita incluyó reuniones intensas de trabajo con el Ministerio de Trabajo y Ministerio de Relaciones Exteriores el 15 de abril. Al día siguiente, se sucedieron encuentros con representantes de Naciones Unidas, de la UIA, con los presidentes de las Cámaras de Diputados y Senadores, y se cerró la jornada con una Conferencia magistral del director general, y con una cena de honor ofrecida por el canciller de la Argentina. El 17 de abril se desarrollaron reuniones con dirigentes de la CGT en las oficinas de la OIT en Argentina, y se llevó adelante una nueva reunión con el ministro de trabajo. El viaje se cerró el 18 de abril con un desayuno de trabajo con el presidente de la nación en Olivos $\frac{33}{3}$. Aunque no se disponen por el momento de más fuentes para analizar con mayor profundidad el impacto y resultados de esta visita, el interés de la OIT en el proceso de transición de la Argentina $-\mathrm{y}$ recíprocamente el interés del gobierno en disponer de instancias de trabajo con esta organización- parece 
reafirmarse en virtud de su extensión, la amplitud y la jerarquía de los funcionarios contactados.

\section{Conclusiones}

En el final del período dictatorial y los primeros tiempos del gobierno democrático, el debate sobre el papel y la dinámica de los trabajadores y el movimiento sindical ocupó un lugar central en la arena política. El presente artículo intentó avanzar sobre un tema poco presente en los numerosos análisis disponibles: la participación de organizaciones internacionales del campo laboral y sindical -muy particularmente la OIT-y su influencia en la forma en la que se saldaron estos debates y en la consolidación de determinadas prácticas, relaciones y formas de organización.

Una primera conclusión de este trabajo -que al incluir nuevas fuentes de archivo antes inaccesibles permite una mirada preliminar y tentativa sobre el primer bienio de la democracia, en el que se tomaron importantes decisiones hacia el futuro- es que la presencia de la OIT, en articulación con la de otras organizaciones como la CIOSL, fue significativa y se manifestó en contactos de diverso tipo, que incluyeron misiones técnicas, contactos entre funcionarios de segunda línea, e incluso contacto directo entre los funcionarios nacionales de primer nivel -entre ellos el presidente de la nación- con las máximas autoridades de la organización. Los elementos presentados aquí indican que la dimensión de las relaciones sindicales internacionales y el papel de algunos organismos y centrales mundiales es relevante para analizar esta historia y, además, tuvo incidencia e impacto en el debate público, ya que ocupó un lugar importante en la prensa, e incidió en el proceso de definición de políticas y de líneas de acción.

Las fuentes examinadas -que sólo permiten relevar una parte de la historia y convocan a un análisis más profundo y extenso a partir del cruce con otros documentos de archivo y testimonios- parecen indicar que el papel de la OIT cambió en forma significativa entre la dictadura y el primer gobierno democrático. Si durante la primera habían existido en la OIT espacios para la denuncia y para la defensa, por parte de algunos sectores, de los derechos sindicales frente a la ofensiva económica, laboral y represiva, canales desde los que se pudo ejercer cierta presión sobre el régimen dictatorial, en la transición a la democracia en cambio, el papel de esta organización parece bastante conservador, reforzando la posición de sectores del movimiento sindical que habían permanecido poco afectados por las políticas regresivas de la dictadura. En instancias cruciales para la reafirmación de la naciente democracia, a algunas de estas figuras que llegaron a declarar no tener ningún conocimiento de las violaciones cometidas contra los derechos humanos, sindicales y laborales se les otorgó un lugar importante y posiciones institucionales que conservaron por décadas, sobre las que luego cimentaron trayectorias de larga duración marcando la historia sindical argentina.

En general, resulta muy destacable en el conjunto de fuentes analizadas la escasa presencia de debate en estos ámbitos entre 1983 y 1985 sobre el legado dictatorial en términos económico-sociales, sindicales y laborales. Esto resulta particularmente llamativo en el caso de las expresiones públicas y los documentos internos de distintos funcionarios de la OIT dada la enorme cantidad de material disponible en el archivo institucional respecto de los efectos de la dictadura en términos laborales y sindicales, como resultado de un importante trabajo acumulado en distintas instancias. Nada de esto aparece puesto en juego en los documentos de archivo disponibles o en ninguna de las coberturas de las instancias de discusión. En cambio, el eje central de la intervención de los funcionarios de la OIT parece centrarse en poner límites a la intervención estatal en el funcionamiento y dinámica del movimiento sindical, sin detenerse a caracterizar los sectores que se consolidan en esta etapa. La ausencia de estas caracterizaciones adquiere relevancia cuando varios de los interlocutores privilegiados resultaron, muy poco tiempo después, críticos de los procesos de judicialización de algunas de las violaciones extremas de derechos de los trabajadores, que estos dirigentes relativizaron o incluso negaron en forma pública y en instancias decisivas. 
En suma, este primer y limitado análisis de las formas de intervención de la OIT durante la dictadura y en los inicios del gobierno democrático debe profundizarse, en vinculación con la reflexión sobre los impactos de un tipo de estructura institucional que, a pesar de fundarse en la dinámica tripartita del diálogo con gobiernos, empresarios y sindicalistas, parece no haber podido encontrar formas de analizar la composición interna, la existencia de tensiones, las confrontaciones y corrientes, los niveles de representatividad y las estructuras institucionales en las cuales estaban insertos cada uno de dichos actores. Tampoco se encuentra en los documentos y fuentes disponibles un análisis fundado de cuestiones clave para esta discusión como la transformación del mercado de trabajo, la distribución del ingreso y la estructura económica y social. De este modo, lo que dado la misión de esta organización debería haber resultado el núcleo de la discusión -el análisis y debate sobre la situación de la estructura económica y social argentina, los derechos y condiciones de vida y de trabajo, y el estado de las estructuras de representación sindical, su dinámica y funcionamientoaparece totalmente ausente en un momento crucial de definición de cuestiones centrales del legado dictatorial. Analizar las conexiones de este proceso con las dinámicas de funcionamiento de los sectores sindicales, que consolidaron su poder de allí en adelante, resulta una línea de investigación promisoria y relevante.

\section{Notas}

1 Este apartado retoma algunas de las conclusiones de la investigación publicada en Basualdo (2010a), basada en fuentes del archivo de la OIT, documentos provenientes de repositorios sindicales, en Argentina y en el exterior del país, fuentes de prensa y publicaciones periódicas.

2 La violencia aplicada desde 1974 y sobre todo durante el gobierno constitucional de María Estela Martínez de Perón tuvo características cualitativamente diferentes a las que vendrían después: hasta 1976, ya que el terrorismo de estado y la desaparición de personas no se encontraban aún institucionalizados. Sobre el estado terrorista y la política de “desaparición de personas” ver Calveiro (1998).

$\underline{3}$ Entre los instrumentos más destacados de legislación laboral de la etapa pueden destacarse: la Ley 21.261 del 24 de marzo de 1976 que suspendió el derecho de huelga; Ley 21.356 de julio de 1976, que prohibió la actividad gremial, es decir asambleas, reuniones, congresos y elecciones, facultando al Ministerio de Trabajo a intervenir y reemplazar dirigentes dentro de los establecimientos fabriles; Ley 21.263 del 24 de marzo de 1976 que eliminó el fuero sindical; Ley 21.259 del 24 de marzo de 1976, que reimplantó la Ley de Residencia, en virtud de la cual todo extranjero sospechoso de atentar contra la "seguridad nacional” podía ser deportado, la Ley 21.400 del 9 de septiembre de 1976, denominada de "Seguridad industrial", que prohibió cualquier medida concertada de acción directa, trabajo a desgano, baja de la producción, etc. (Gallitelli y Thompson, 1990, pp. 28-30, entre otros).

4 Sobre el caso 809, ver por ejemplo el Reporte 157 del Comité de Libertad Sindical (CFA), GB. 199 (Febrero-Marzo 1976), pp. 14-16. Sobre el caso 836, ver por ejemplo el Reporte 160 del CFA, GB. 201 (Noviembre, 1976), p. 51.

$\underline{5}$ Aunque la Federación Sindical Mundial apoyaba las demandas referidas a la recuperación de los derechos laborales en Argentina, esta organización, alineada con la URSS y la posición de los Partidos Comunistas tenía una posición contradictoria respecto de la dictadura, muy influida por las fuertes relaciones comerciales de la Argentina con la Unión Soviética y la posición del PCA que creía que la Junta que había tomado el poder tenía una posición "moderada" dentro del arco de las fuerzas armadas. Por lo tanto, aunque denunciaron la desaparición de trabajadores, atribuían muchas de ellas a bandas de ultraderecha y exigían al gobierno (el directo responsable), que frenaran su accionar. Eventualmente, cuando la responsabilidad directa 
de las fuerzas armadas en estas graves violaciones se hizo innegable, la FSM endureció su posición respecto de la dictadura. Ver el comunicado de prensa firmado por la FSM "Stopper l'action des groupes terroristes en Argentine!” (Octubre 1, 1976) en el archivo de la CGT-FSM, Francia.

6 Ver "En Ginebra los asuntos laborales se desplazan”, La Opinión (Mayo 30, 1976); “La OIT, conflictuada”, Clarín (Junio 4, 1977); "Las pugnas políticas en la OIT, Clarín (Junio 7, 1977); "Futuro incierto para la OIT”, Clarín (Junio 21, 1977); “Decidió reducir sus actividades la OIT”, La Prensa, (Noviembre 2, 1977); “Kissinger y el retiro de la OIT”, La Opinión (Noviembre 22, 1977), entre otros. La decisión tomada por James Carter, entonces Presidente de Estados Unidos de retirar al país de la OIT fue analizada y discutida muy extensamente en la prensa argentina.

$\underline{7}$ En noviembre de 1979, luego de una primera etapa de profundas transformaciones de la dinámica sindical y de los derechos de los trabajadores en los hechos y en la normativa en un marco de represión de una intensidad extrema, la dictadura había promulgado una nueva Ley de Asociaciones Profesionales, la Ley 22.105. A pesar de presentar una gran cantidad de aspectos limitantes de la acción sindical, restrictivos de los derechos laborales y de organización, esta norma no apuntaba a desmembrar la estructura de las organizaciones sindicales en sí, a excepción de la Confederación General del Trabajo, cuya existencia no contemplaba (Campos, 2009; Zorzoli, 2015). En cambio, constituía una pieza más en un proyecto de transformación estructural de las funciones de las organizaciones sindicales y su conversión en actores subordinados, que debe ser comprendida en el marco del conjunto de las transformaciones estructurales y laborales, así como de las políticas represivas llevadas adelante en esta etapa. A partir de este marco normativo, las organizaciones debían adecuar sus estatutos a las nuevas prescripciones legales como requisito previo para alcanzar su normalización definitiva.

8 Nota del diario Visión, 16 de enero de 1984 en Cabinet files, carpeta "2 1-2-1 (J1) Relations with Argentina July 1974- 31-12-85”, Archivo OIT.

9 Nota del diario La Nación, el 30 de enero de 1984, en Cabinet files, carpeta "2 1-2-1 (J1) Relations with Argentina July 1974- 31-12-85”, Archivo OIT.

10 Nota de diario La Nación, 30 de enero de 1984, en Cabinet files, carpeta "2 1-2-1 (J1) Relations with Argentina July 1974- 31-12-85", Archivo OIT.

11 Nota de diario La Nación, 5 de febrero de 1984, en Cabinet files, carpeta "2 1-2-1 (J1) Relations with Argentina July 1974- 31-12-85”, Archivo OIT.

12 Nota diario La Razón, Buenos Aires, 4 de febrero de 1984, en Cabinet files, carpeta "2 1-2-1 (J1) Relations with Argentina July 1974- 31-12-85”, Archivo OIT.

13 Nota en diario Tiempo Argentino, 4 de febrero de 1984, en Cabinet files, carpeta "2 1-2-1 (J1) Relations with Argentina July 1974- 31-12-85”, Archivo OIT.

14 Carta de Raúl Alfonsín a Francis Blanchard, fechada el 15 de febrero de 1984, Cabinet files, carpeta "2 12-1 (J1) Relations with Argentina July 1974- 31-12-85”, Archivo OIT.

15 Carta de Francis Blanchard a Raúl Alfonsín fechada el 2 de marzo de 1984, Cabinet files, carpeta "2 1-2-1 (J1) Relations with Argentina July 1974- 31-12-85”, Archivo OIT.

16 Diario El Día, 27 de febrero de 1984 incluida en Cabinet files, carpeta "2 1-2-1 (J1) Relations with Argentina July 1974- 31-12-85”, Archivo OIT.

17 Para una síntesis de la trayectoria de Ramón A. Baldassini consultar el Expte. no 5662/11 Proyecto de Decreto s/petición presentada por vecinos de la ciudad de La Paz para la declaración de ciudadano ilustre en 
la provincia de Entre Ríos, Argentina, citado en: https://lanotadigital.com.ar/2012/04/24/declaran-ciudadanoilustre-a-baldassini-miembro-de-la-cgt/ Ramón Baldassini es el sindicalista argentino con mayor permanencia como Secretario General de su sindicato por un total de 54 años, entre 1963 y 2017, puesto que abandonó para integrar el sector empresarial al ser designado integrante del directorio de la empresa Correo Argentino (“Adiós al sindicalista récord: Ramón Baldassini deja el poder tras 54 años”, diario La Nación, 9 de mayo de 2017).

18 Artículo "La OIT nominó a Baldassini en un ente permanente” del 6 de marzo de 1984, en Cabinet files, carpeta “2 1-2-1 (J1) Relations with Argentina July 1974- 31-12-85”, Archivo OIT. La designación informada por esta fuente de prensa está confirmada por la documentación oficial de la propia OIT: http://staging.ilo.org/public/libdoc/ilo/1985/85B09 156 span.pdf

19 Artículo "Críticas de dirigentes del CIOSL a la Ley Sindical” en diario La Prensa del 9 de marzo de 1984, Cabinet files, carpeta “2 1-2-1 (J1) Relations with Argentina July 1974- 31-12-85”, Archivo OIT.

$\underline{20}$ Nota en diario Tiempo Argentino, martes 13 de marzo de 1984, en Cabinet files, carpeta "2 1-2-1 (J1) Relations with Argentina July 1974- 31-12-85”, Archivo OIT.

$\underline{21}$ Nota en diario Tiempo Argentino, martes 13 de marzo de 1984, en Cabinet files, carpeta "2 1-2-1 (J1) Relations with Argentina July 1974- 31-12-85”, Archivo OIT.

$\underline{22}$ Ver por ejemplo nota en diario La Voz, 17 de marzo de 1984, "El rechazo de la Ley Sindical plantea un dilema al gobierno” en Cabinet files, carpeta "2 1-2-1 (J1) Relations with Argentina July 1974- 31-12-85”, Archivo OIT.

23 Cable de Julio Galer a Francis Blanchard, fechado el 13 de abril de 1984, en Cabinet files, carpeta "2 1-21 (J1) Relations with Argentina July 1974- 31-12-85”, Archivo OIT.

$\underline{24}$ Cable de Francis Blanchard a Raúl Alfonsín con fecha 2-5-1984 en Cabinet files, carpeta "2 1-2-1 (J1) Relations with Argentina July 1974- 31-12-85”, Archivo OIT.

$\underline{25}$ Nota de Diario Popular, Sábado 19 de Mayo de 1984, en Cabinet files, carpeta "2 1-2-1 (J1) Relations with Argentina July 1974- 31-12-85”, Archivo OIT.

26 Nota de diario La Prensa, 23 de mayo de 1984, en Cabinet files, carpeta "2 1-2-1 (J1) Relations with Argentina July 1974- 31-12-85”, Archivo OIT.

$\underline{27}$ Nota "La próxima será una semana de definiciones en los problemas que preocupan a los gremialistas" en diario La Razón, 24 de mayo de 1984, en Cabinet files, carpeta "2 1-2-1 (J1) Relations with Argentina July 1974- 31-12-85”, Archivo OIT.

$\underline{28}$ Informe sobre Argentina preparado para la misión de 1984, en Cabinet files, carpeta "2 1-2-1 (J1) Relations with Argentina July 1974- 31-12-85”, Archivo OIT.

$\underline{29}$ Informe sobre Argentina preparado para la misión de 1984, en Cabinet files, carpeta "2 1-2-1 (J1) Relations with Argentina July 1974- 31-12-85”, Archivo OIT.

$\underline{30}$ Nota "Autodeterminación de los trabajadores. El Presidente recibió al experto de la OIT que asesora al gobierno" en diario Tiempo Argentino, viernes 1 de junio de 1984, en Cabinet files, carpeta "2 1-2-1 (J1) Relations with Argentina July 1974- 31-12-85”, Archivo OIT.

$\underline{31}$ Nota "Autodeterminación de los trabajadores. El Presidente recibió al experto de la OIT que asesora al gobierno" en diario Tiempo Argentino, viernes 1 de junio de 1984, en Cabinet files, carpeta "2 1-2-1 (J1) Relations with Argentina July 1974- 31-12-85”, Archivo OIT. 
32 Documento "Rapport de misión. Projet de recherche sur les activités de bien-etre des syndicats dans le paysen développement, Argentina 5-9 de novembre 1984” elaborado por R. de Grazia, en Cabinet files, carpeta “2 1-2-1 (J1) Relations with Argentina July 1974- 31-12-85”, Archivo OIT.

33 Documento "Programa en la Argentina del Señor Director General de la Organización Internacional del Trabajo Dr. Francis Blanchard” elaborado por el Ministerio de Trabajo y Seguridad Social de la Nación Argentina, en Cabinet files, carpeta "2 1-2-1 (J1) Relations with Argentina July 1974- 31-12-85”, Archivo OIT.

\section{Bibliografía}

AEyT de FLACSO, CELS, PVJ y SDH (2015). Responsabilidad empresarial en delitos de lesa humanidad: represión a trabajadores durante el Terrorismo de Estado, Editorial Infojus (segunda edición Editorial de la Universidad de Misiones 2016).

Aruguete, E. (2006). Lucha política y conflicto de clases en la posdictadura. Límites a la constitución de alianzas policlasistas durante la administración Alfonsín. En A. Pucciarelli (comp), Economía y política durante el gobierno de Alfonsín. El poder de la democracia o la democracia del poder, pp. 413-460 Buenos Aires: Siglo Veintiuno.

Azpiazu, D., Basualdo, V. y Schorr, M. (2010). La industria y el sindicalismo de base en la Argentina. Buenos Aires: Cara o Ceca.

Basualdo, E. (2006). Estudios de historia económica argentina. Desde mediados del siglo XX a la actualidad. Buenos Aires: Editorial Siglo Veintiuno.

Basualdo, V. (2006). Complicidad patronal-militar en la última dictadura argentina. Los casos de Acindar, Astarsa, Dálmine Siderca, Ford, Ledesma y Mercedes Benz. En Suplemento especial de Engranajes a 30 años del golpe militar, FETIA-CTA, marzo de 2006 disponible en: http://www.riehr.com.ar/detalleInv.php? $\underline{\mathrm{id}=7}$.

Basualdo, V. (2010a). The ILO and the Argentine dictatorship, 1976-1983. En J. Van Daele, M. Rodríguez García, G. Van Goethem, M. Van der Linden (eds.), ILO Histories. Essays on the International Labour Organization and Its Impact on the World During the Twentieth Century, pp. 401-423. Berlín y Nueva York: Peter Lang.

Basualdo, V. con la colaboración de Ivonne Barragán y Florencia Rodríguez (2010b). Dossier: La clase trabajadora durante la última dictadura militar argentina (1976-1983): apuntes para una discusión sobre la resistencia obrera, Comisión Provincial por la Memoria.

Basualdo, V. (2010c). Labor and Structural Change: Shop-floor Organization and Militancy in Argentine Industrial Factories (1943--1983), Ph.D dissertation, Columbia University.

Calveiro, P. (1988). Poder y desaparición. Los campos de concentración en Argentina. Buenos Aires: Editorial Colihue.

Campos, L. (2009). Estado y sindicatos: un análisis de sus relaciones a partir de los mecanismos de regulación y la conformación de la estructura sindical en la Argentina (1943-1983) (Tesis de Maestría), FLACSO, Argentina.

Galante, D. (2015). El "Juicio a las juntas" en la escena política argentina. Revista Lucha Armada en la Argentina: Año 10, Anuario octubre 2014 / Agosto 2015, Disponible en: http://historiapolitica.com/datos/biblioteca/ochentas galante.pdf 
Gallitelli, B. y Thompson, A. (1990). La política laboral en la Argentina del "Proceso”. En M. Barrera y G. Fallabella, Sindicatos bajo regímenes militares. Argentina, Brasil, Chile, pp. 23-67. Santiago de Chile: CESNaciones Unidas.

Gargarella, R. (2010). Democracia y derechos en los años de Raúl Alfonsín. En R. Gargarella, M. V. Murillo y M. Pecheny (comps.), Discutir Alfonsín. Buenos Aires: Siglo Veintiuno.

Gaudio, R. y Domeniconi, H. (1986). Las primeras elecciones sindicales en la transición democrática. Revista Desarrollo Económico, 26(103), 423-454.

Gaudio, R. y Thompson, A. (1990). Sindicalismo peronista/gobierno radical. Los años de Alfonsín. Buenos Aires: Fundación Friedrich Ebert.

Gordillo, M. B. (2013). Normalización y democratización sindical: repensando los '80. Revista Desarrollo Económico, 53 (209-210), pp. 143-167.

Izaguirre, I. (2009), Las luchas obreras y el genocidio en la Argentina. En I. Izaguirre (dir), Lucha de clases, guerra civil y genocidio en la Argentina, 1973-1983, , pp. 245-282. Buenos Aires: Eudeba.

Murillo, M. V. (2010). ¿Las corporaciones o los votos? En R. Gargarella, M. V. Murillo y M. Pecheny (comps.), Discutir Alfonsín, pp- 139-160. Buenos Aires: Siglo Veintiuno.

Palomino, H. (2005). Los cambios en el mundo del trabajo y los dilemas sociales. En J. Suriano (dir.), Dictadura y democracia (1976-2001) (pp. 377-389). Buenos Aires: Sudamericana.

Senén González, S. y Bosoer, F. (1993). La trama gremial 1983-1989: crónica y testimonios. Buenos Aires: Corregidor.

Verbitsky, H. y Bohoslavsky, J. P. (2013). Cuentas pendientes. Los cómplices económicos de la dictadura. Buenos Aires: Siglo Veintiuno.

Zorzoli, L. (2015). La normativa sindical entre la dictadura y el alfonsinismo, propuesta de sistematización. En A. Schneider y P. Ghigliani (eds.), Clase obrera, sindicatos y Estado. Argentina (1955-2010). Buenos Aires, Imago Mundi.

Zorzoli, L. (2016). Operativo Ginebra. La dirigencia sindical ante la instalación internacional de la dictadura militar. Revista Archivos, Año IV, 8, 13-32. 